\title{
La autopublicación en las bibliotecas: revisión sistemática de la literatura
}

\author{
Almudena Mangas-Vega \\ Universidad de Salamanca, España \\ almumvega@usal.es \\ Javier Merchán Sánchez-Jara \\ Universidad de Salamanca, España \\ javiermerchan@usal.es

\section{Alberto Ramos Alonso} \\ Universidad de Salamanca, España \\ albertoinfodocu@gmail.com
}

Cita sugerida: Mangas-Vega, A.; Merchán Sánchez-Jara, J y Alonso, A. R. (2018). La autopublicación en las bibliotecas: revisión sistemática de la literatura. Palabra Clave (La Plata), 7(2), e047. https://doi.org/10.24215/18539912e047 


\section{La autopublicación en las bibliotecas: revisión sistemática de la literatura}

\section{Self-publishing in libraries: sistematic literature review}

Almudena Mangas-Vega

Universidad de Salamanca, España

almumvega@usal.es

Javier Merchán Sánchez-Jara

Universidad de Salamanca, España

javiermerchan@usal.es

Alberto Ramos Alonso

Universidad de Salamanca, España

albertoinfodocu@gmail.com

\section{RESUMEN:}

La autopublicación no es un fenómeno que tenga su nacimiento en época reciente, pero ha encontrado en lo digital un catalizador para ganar un enorme crecimiento en los últimos años. El trabajo estudia, a través de una revisión sistemática de literatura (en inglés, SLR), si este fenómeno ha penetrado en la comunidad bibliotecaria y de qué modo lo ha hecho. Los resultados muestran un tema que ha tomado gran relevancia, aunque con una marcada tendencia geográfica de carácter anglosajón. La autopublicación aparece como elemento de la colección ofrecida y como servicio a los usuarios, y en todos los casos se habla de experiencias positivas. El resto del mundo no anglosajón se muestra conservador en este tema, pero tiene ante sí una nueva línea de investigación y el bagaje de las experiencias de estos trabajos para satisfacer de la mejor manera posible esta nueva necesidad de sus usuarios.

Palabras Clave: Autopublicación, Bibliotecas, Revisión Sistema Literatura.

\section{Abstract:}

Self-publishing is not a phenomenon that has its birth in recent times but has found in digital a catalyst to gain enormous growth in last years. The work studies through a systematic review of literature (in English, SLR), if this phenomenon has penetrated the library community and in what way it has done so. The results show a topic that has taken high relevance, although with a marked geographical tendency of Anglo-Saxon character. Self-publishing appears as an element of the collection offered and as a service to users, and in all cases, we talk about positive experiences. The rest of the non-Anglo-Saxon world is conservative on this issue but has before it a new line of research and the background of the experiences of these works to satisfy this further need of its users in the best way possible.

KEYWORDS: Self-publishing, Libraries, Systematic Literature Review.

\section{INTRODUCCIÓN}

A lo largo de la historia de la cultura occidental, el libro se ha conformado como el instrumento arquetípico para vehicular la fijación y transmisión del conocimiento y la creación literaria. Su desarrollo y perfeccionamiento como objeto cultural ha implicado la generación de un ecosistema en el que han ido apareciendo y desapareciendo distintos agentes, prácticas, necesidades o instituciones que conforman parte esencial de este universo particular. A lo largo de esta evolución se inscriben algunos de los hitos más significativos para el desarrollo cultural, social y económico de la humanidad. El surgimiento de la imprenta, o la inmersión de los procesos de edición y publicación en el entorno digital, son solo algunos de los hechos que han sumido al libro, y por extensión al mundo de la cultura y la comunicación, en un periodo revolucionario (Cordón-García, 2011).

Una de las cualidades inherentes a todo periodo revolucionario es la constatación de la disparidad de criterios desde la que se contempla el fenómeno, así como la declaración de multitud de opiniones, muchas 
de ellas diametralmente opuestas, sobre todo aquello que es considerado novedoso, o entra en conflicto de manera directa con respecto a las prácticas y el conjunto de saberes establecidos hasta el momento.

La irrupción de los libros digitales en el pasado siglo XX es un ejemplo muy significativo para ilustrar alguna de estas cuestiones. La aplicación de las nuevas tecnologías digitales (o, mejor dicho, su implantación y normalización a nivel social) en el ámbito de la edición y publicación de textos, relegó al ostracismo la anterior etapa "revolucionaria": la "ola transformadora" del mundo del libro que representó el desarrollo y uso del offset, abaratando el coste de los libros y propiciando el fenómeno global del libro del bolsillo. Todos estos fenómenos disruptivos han ido permeando el universo social y cultural, a diferente velocidad dependiendo de las circunstancias históricas de cada territorio (EDI-RED, s.f.),pero en todos los casos ha provocado un aumento sin precedentes en el número de agentes y elementos implicados en la producción, publicación y distribución de libro.

Si bien es cierto que el libro digital encontró una férrea oposición por gran parte del mundo del libro y la lectura, tratando de mostrarlo como opuesto al libro en papel y vaticinando su inminente final allá por 2011 (Furtado, 2012), finalmente el libro digital ha acabado normalizándose en la sociedad modificando nuestro comportamiento como lectores, consumidores, educadores, y en definitiva, la manera en que nos relacionamos con el texto como referente en el ámbito del conocimiento y la cultura (Gómez-Díaz, GarcíaRodríguez y Cordón-García, 2015).

El concepto, la dimensión y las prácticas relacionadas con el proceso de publicación, son otros de los elementos centrales que se han visto afectados con mayor intensidad y nivel de influencia en el ámbito de la producción y la diseminación de los materiales textuales. Conceptualmente la idea de publicación ha ido modulando su significación de acuerdo a un elenco de necesidades y condicionantes socioeconómicos propios de cada época y cultura. En los albores del desarrollo de la imprenta, el concepto estaba internamente ligado a todo el conjunto de gestiones y requerimientos necesarios para incorporar a la obra las pertinentes licencias de impresión. Este referente evoluciona, en épocas posteriores, hacia la idea de la publicación como la puesta a disposición al público de la información y las ideas propias de una determinada cultura, así como los procedimientos, medios y técnicas empleados para lograr este fin. Finalmente, en las épocas más recientes, esta idea central de la publicación como procedimiento de "dar a conocer", ha ido incorporando otros matices y requerimientos, generalmente relacionados con el al acto de juzgar y controlar la calidad e idoneidad del objeto producido de acuerdo a consideraciones de carácter formal, de calidad, o incluso ético y moral; en este sentido, el proceso de publicación consiste, esencialmente, en decidir qué documento debe de ser producido, cómo, y para qué propósito (Saffle, 2010).

Algo similar ha ocurrido con la autopublicación. Existen registros de libros autopublicados desde la antigüedad, como por ejemplo el caso del médico romano Galeno, que en sus obras Peri ton idion biblion y Peri tes taxeos ton idion biblion (alrededor del año 190 d. C.) quiso dejar patente en el título que se trataba de autopublicación.

También este fenómeno sufrió críticas, e incluso hoy hay quien le sigue aplicando la categoría de "publicación de vanidad" aludiendo a la falta de calidad de los escritos. Sin embargo, cada vez son más y más los libros autopublicados, sus lectores y los autores (anónimos o de renombre) que autopublican (MangasVega, 2016a)

Está siendo, además, una gran oportunidad para libros destinados a nichos reducidos de lectores, o el resurgir de obras que con el paso del tiempo las editoriales decidieron dejar de editar y que ahora sus autores, recuperados los derechos, autopublican para darles una nueva vida.

En este escenario, existen numerosas opciones y herramientas al alcance de los autores. Si definimos autopublicación como el proceso en el que el autor tiene el control sobre todas las tareas a realizar -aun cuando contrate a terceros para su ejecución (Mangas-Vega y Gómez-Díaz, 2015)-, la elección de la empresa o institución de apoyo se convierte en el paso más importante. Muchas de estas herramientas llegan desde las 
editoriales tradicionales que, ante la bajada de lectores en sus autores consagrados habituales, han encontrado en la autopublicación la manera de salir a flote en la reciente época de crisis (Rodríguez Marcos, 2016).

No es de extrañar, pues, que la autopublicación tuviera un incremento del 436\% entre 2008 y 2013 (Bowker, 2014). Y es habitual también ver este tipo de libros entre los más consumidos en plataformas como Amazon o Smaswords (Cordón-García, 2016).

Sin embargo, ha habido un elemento constante a lo largo de la historia del libro, un agente que ha sabido adaptarse paulatinamente a los cambios, con mayor o menor rapidez y fortuna, pero sin entrar en controversias y sin tomar parte más allá de la de buscar el bien de sus usuarios. Es la biblioteca.

No resulta ilógico si pensamos que su fundación y desarrollo ha tenido siempre al libro como elemento indispensable y a los usuarios como pilares fundamentales. Usuarios que actualmente ya no son meros lectores, ya que ahora una gran mayoría son comunicadores (a través de Internet) y muchos son también escritores. No en vano, no existe escritura sin lectura.

Y de la misma manera, la biblioteca parece, a priori, el lugar natural de la autopublicación, ya sea para su lectura o su escritura.

Desde la aparición de las nuevas tecnologías, la biblioteca, en esa continua búsqueda de adaptación a las necesidades del usuario, ha tratado de amoldar sus servicios y productos a sus demandas. Esto ha implicado reorganización de espacios, revisión de colecciones, nuevos servicios, etc. La pregunta, pues, es: ¿Es la autopublicación uno más de los giros de la biblioteca actual? ¿En qué sentido se está trabajando con la autopublicación en las bibliotecas? ¿Hasta qué grado ha penetrado este fenómeno en el mundo bibliotecario? El artículo trata de responder a estas preguntas a través de una revisión de los trabajos más relevantes publicados sobre el tema.

Para ello se realizará una revisión sistemática de literatura (SLR, de su nombre en inglés, Systematic Literature Review), uno de los tipos de revisiones más potentes actualmente y que está dando buenos resultados también en Ciencias Sociales y Humanidades.

\section{Metodología}

La Revisión Sistemática de Literatura (en inglés, SLR) es una metodología surgida en el campo de la medicina con la intención de proveer a los especialistas de un sistema que permitiera de forma rápida y eficaz hacerse con los trabajos más relevantes en un tema (Ramírez, 2007). Sin embargo, lleva ya varias décadas demostrando ser un sistema de revisión de la literatura potente y con muy buenos resultados en diferentes disciplinas (Petticrew y Roberts, 2008; Kitchenham, 2004). Es por ello que ha sido elegida como metodología a seguir en este caso.

Teniendo en cuenta la especificidad y singularidad de la materia buscada, los pasos a realizar han sido los siguientes:

Definición de los objetivos y/o preguntas de investigación

- Se definieron como preguntas de investigación las siguientes:

- RQ1: Según las iniciativas y proyectos sobre el tema, ¿cuál está siendo el papel de la biblioteca respecto a la autopublicación?

- RQ2: ¿El tema tiene alguna distribución geográfica o tipológica destacable?

- RQ3: Según las iniciativas y proyectos en bibliotecas académicas, ¿cuál está siendo la penetración del tema dentro de la comunidad científica?

Definición de los criterios de inclusión de los trabajos en la SLR 
- Atendiendo al alcance de la revisión a cuestiones técnicas que facilitasen su desarrollo, se definieron como criterios de inclusión los siguientes:

- IC1: Los trabajos tratan expresamente el papel de la biblioteca en el desarrollo del fenómeno de la autopublicación a través de sus usuarios, o recogen iniciativas o estrategias que lo mencionen.

- IC2: Los trabajos están escritos en español, inglés, francés, italiano o portugués.

- IC3: Los trabajos han sufrido revisión por pares, o están incluidos en Jornadas o Conferencias Técnicas que cuenten con un sistema de revisión científica por pares.

- IC4: Se tiene acceso al texto completo del trabajo.

Definición de los criterios de exclusión de los trabajos en la SLR.

- De nuevo, atendiendo a cuestiones prácticas, se definieron los siguientes criterios de exclusión:

- EC1: Los trabajos hablan de las nuevas funciones de las bibliotecas y de la autopublicación, pero no de proyectos o iniciativas que relacionen ambos temas.

- EC2: Los trabajos no están escritos en español, inglés, francés, italiano o portugués

- EC3: Los trabajos no han sufrido sistema de revisión por pares.

- EC4: No se tiene acceso al texto completo del trabajo.

Selección de las bases de datos y fuentes de información: en este caso, fue necesario definir como criterio de selección obligatorio el libre acceso a los recursos durante todo el proceso de desarrollo del trabajo.

- Como recursos de información fueron elegidos los siguientes:

- Bases de datos científicas de carácter general: WOS (Thomson Reuters), SCOPUS (Elsevier)

- Bases de datos científicas especializadas en el área de Información y Documentación: LISA (ProQuest) y LISTA (EBSCOhost)

Definición de los términos y las cadenas de búsqueda en los recursos de información:

- A la hora de seleccionar los términos, se ha tenido en cuenta el carácter internacional de la búsqueda de información, por lo que los términos se han seleccionado en inglés. Los términos elegidos finalmente han sido self-publishing (con diferentes grafías) para referirse a la autopublicación y library (en singular y plural) para abarcar las bibliotecas: (self-publising OR self publishing OR selfpublisinhg OR auto-publisihing) AND (libary OR libraries).

La cadena de búsqueda general resultante ha sido la siguiente:

- [(“stake-holder” OR “” OR “” OR “”) AND (“libary” OR “) AND (“self-publishing” OR "self publishing" OR "selfpublishing”)]

Hay que indicar que, debido a las diferencias en los campos de búsqueda ofrecidos por los distintos recursos, esta cadena ha sufrido alguna ligera modificación según el recurso, tratando en todo momento de no distorsionar la búsqueda.

Definición de los límites de búsqueda

- Se han diseñado búsquedas en base a:

- Rango temporal: el tema buscado es de una aparición reciente, por lo que se ha limitado la búsqueda a trabajos posteriores a 2005 ya que, según muchos autores, es en esa fecha cuando la aparición de nuevos dispositivos electrónicos fomenta el auge de los libros electrónicos, 
que sufren un rápido aumento en número y lectores (Cordón García, Gómez Díaz y Alonso Arévalo, 2011).

- Límite por campos: Topic (en el caso de WOS) y Article title, abstract and keywords (en el resto de bases de datos).

Fases de la revisión

- La revisión, siguiendo modelos utilizados anteriormente en otras materias de Ciencias Sociales y Humanidades (Mangas-Vega, 2016b), ha consistido en un ciclo de la siguiente sucesión de fases:

- Fase 1: Búsqueda (definición de la cadena de búsqueda y aplicación de la misma).

- Fase 2: Eliminación de duplicados a través de software de gestión bibliográfica.

- Fase 3: Revisión de los títulos y resúmenes de los trabajos y aplicación de los criterios de inclusión y exclusión definidos previamente.

- Fase 4: Revisión del texto completo de los trabajos restantes y nueva aplicación de criterios de inclusión y exclusión definidos previamente.

- Fase 5: En caso necesario, inclusión de trabajos relevantes que no hayan sido recuperados en la búsqueda.

- Fase 6: Repetición del proceso para asegurar su fiabilidad.

Evaluación de la calidad de los resultados.

- En el caso de que los elementos recuperados sean demasiado numerosos para poder abordar su revisión, se procederá a valorar la inclusión o no de cada trabajo en base a la posición en JCR o SJR de la revista que lo haya publicado.

Cada uno de los pasos dados ha implicado una autoevaluación de la propia metodología en base a los resultados parciales obtenidos, y se han realizado las correcciones pertinentes para el buen desarrollo de la revisión y la consecución de los objetivos definidos a través de las preguntas de investigación. 


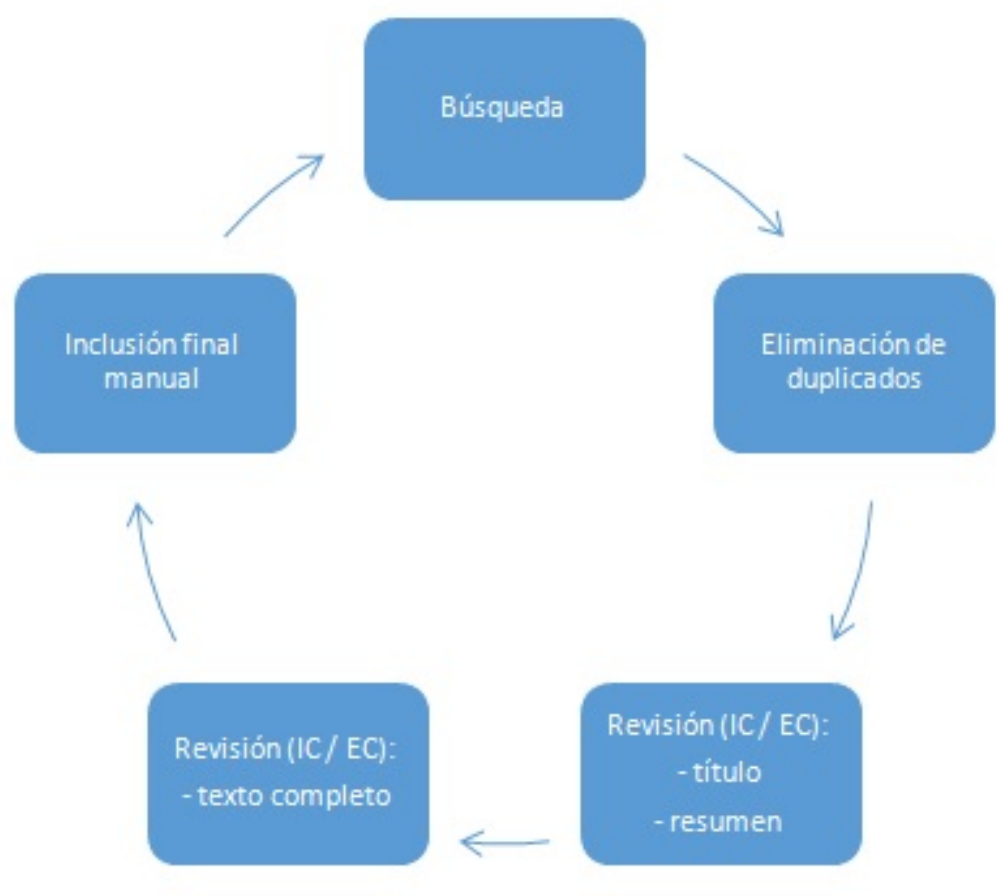

FIGURA 1

Esquema del proceso de la Revisión Sistemática de Literatura realizada

Elaboración propia.

\section{Resultados y discusión}

La metodología utilizada, Revisión Sistemática de Literatura, ha resultado totalmente satisfactoria. A tenor de los resultados obtenidos y del patrón de referencias observado en los mismos, el proceso ha permitido reducir significativamente la cantidad de elementos a revisar sin la pérdida de ninguno relevante. Se ha obtenido como producto final una serie abarcable, lógica y pertinente de trabajos, que ha permitido generar una revisión completa y fundamentada.

En la tabla 1 se muestra el proceso y el número de artículos resultante de cada fase.

\begin{tabular}{|c|c|c|c|c|c|c|}
\hline \multicolumn{2}{|c|}{ Proceso SLR } & WoS & Scopus & LISA & LISTA & Total \\
\hline Fase 1 & Resultados & 36 & 31 & 414 & 177 & 658 \\
\hline (IC2) & 1-Filtro idioma & 35 & 30 & 402 & 174 & 641 \\
\hline (IC3) & $\begin{array}{l}\text { 2-Filtro peer } \\
\text { review }\end{array}$ & 35 & 30 & 270 & 70 & 405 \\
\hline $\begin{array}{l}\text { Fase } 2 \\
\text { Fase } 3 \\
\text { (IC1) } \\
\text { (realizadas juntas } \\
\text { por motivos } \\
\text { técnicos) }\end{array}$ & $\begin{array}{l}\text { 3-Filtro temática y } \\
\text { duplicados }\end{array}$ & 10 & 8 & 20 & 9 & 47 \\
\hline Fase 4 & Filtro acceso & $-\cdots$ & ---- & $-\cdots$ & ---- & 21 \\
\hline $\begin{array}{l}\text { Eliminación de } \\
\text { duplicados }\end{array}$ & Duplicados & & & & & 18 \\
\hline Fase 5 & Inclusión & & & & & 19 \\
\hline Resultado & & & & & & 19 \\
\hline
\end{tabular}

TABLA 1

Proceso de SLR y número de resultados obtenidos en cada fase 
Los trabajos obtenidos como resultado de la aplicación de la metodología SLR y revisados finalmente han sido los que se detallan en la tabla 2.

\begin{tabular}{|c|c|}
\hline Título & Autores \\
\hline $\begin{array}{l}\text { Role changing for librarians in the } \\
\text { new information technology era }\end{array}$ & $\begin{array}{l}\text { Hao-Chang Sun, Kuan-nien Chen, } \\
\text { Chishu Tseng, Wen-Hui Tsai }\end{array}$ \\
\hline $\begin{array}{l}\text { Electronic books: their integration into library } \\
\text { and information centers }\end{array}$ & Siriginidi Subba Rao, \\
\hline $\begin{array}{l}\text { Support for alternative publishing by public } \\
\text { libraries in Scotland }\end{array}$ & Angela Brookens, Alan Poulter \\
\hline $\begin{array}{l}\text { The Role of Public Libraries in Self- } \\
\text { Publishing: Investigating Author and } \\
\text { Librarian Perspectives }\end{array}$ & Heather Moulaison Sandy \\
\hline $\begin{array}{l}\text { Self-Published Books: An Empirical } \\
\text { "Snapshot" }\end{array}$ & $\begin{array}{l}\text { Jana Bradley, Bruce Fulton and } \\
\text { Marlene Helm4 }\end{array}$ \\
\hline $\begin{array}{l}\text { Small Press and Self-Published Books: A } \\
\text { Collection Development Dilemma }\end{array}$ & Matt Pacer \\
\hline Self-Publishing: Breaking Down Barriers & Bob Nardini y Janice Schnell \\
\hline Self-Publishing: A Bibliographic Essay & Joseph D. Grobelny \\
\hline $\begin{array}{l}\text { Self-Publish or Traditional? My Experience } \\
\text { with Books for Librarians }\end{array}$ & Walt Crawford \\
\hline The self-publishing phenomenon and libraries & Juris Dilevko, Keren Dali \\
\hline $\begin{array}{l}\text { Public Libraries as Publishers: Critical } \\
\text { Opportunity }\end{array}$ & Kathryn M. Conra \\
\hline $\begin{array}{l}\text { Beyond traditional publishing models An } \\
\text { examination of the relationships between } \\
\text { authors, readers, and publishers }\end{array}$ & Jen (J.L.) Pecoskie y Heather Hill \\
\hline $\begin{array}{l}\text { E-publishing in libraries: the [Digital] } \\
\text { preservation imperative }\end{array}$ & $\begin{array}{l}\text { Heather Lea Moulaison and A. J. } \\
\text { Million }\end{array}$ \\
\hline $\begin{array}{l}\text { OCLC Systems \& Services: International } \\
\text { digital library perspectives }\end{array}$ & Laura Cruz y Rachel Flemming \\
\hline $\begin{array}{l}\text { Self-Publishing and Collection Development. } \\
\text { Opportunities and Challenges for Libraries }\end{array}$ & Robert P. Holley (Ed.) \\
\hline $\begin{array}{l}\text { Several Factors of Library Publishing Services } \\
\text { Facilitate Scholarly Communication Functions }\end{array}$ & Leslie Bussert \\
\hline $\begin{array}{l}\text { Exploring How Library Publishing Services } \\
\text { Facilitate Scholarly Communication }\end{array}$ & Ji-Hong Park and Jiyoung Shim \\
\hline The Role of Self-Publishing in Libraries & Laura Dawson \\
\hline $\begin{array}{l}\text { Self-publishing and musicology: historical } \\
\text { perspectives, problems, and } \\
\text { possibilities }\end{array}$ & Michael Saffle \\
\hline
\end{tabular}

TABLA 2

Trabajos obtenidos como resultado de la revisión sistemática de literatura

Antes de profundizar en los datos obtenidos y su discusión, cabe destacar que, si bien no entra dentro del propósito de este trabajo realizar un mapping de los mismos, hay elementos que sí han necesitado un análisis cuantitativo para dar respuesta a las preguntas de investigación.

Uno de los primeros datos observados es que se trata de un tópico geográficamente muy concentrado: el $74 \%$ de los trabajos revisados tienen a su autor principal afiliado en USA, porcentaje que aumenta hasta el 95\% si se tiene en cuenta la afiliación de todos los autores y no sólo la del autor principal. 


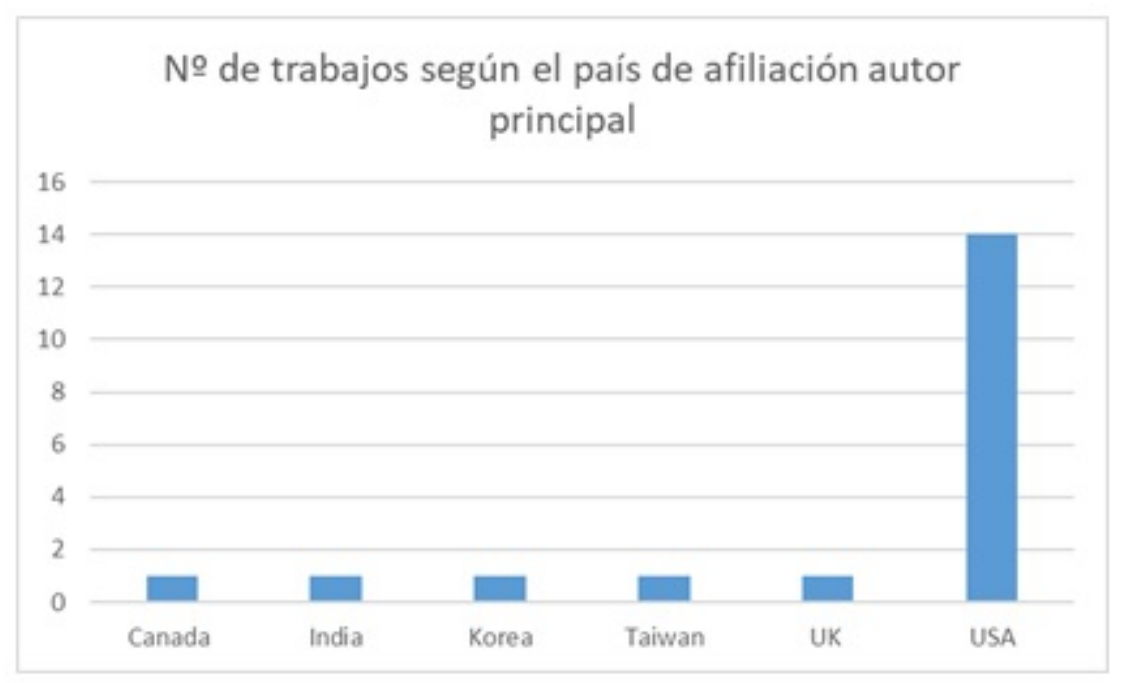

FIGURA 2

Cantidad de trabajos por país de afiliación del autor principal

Elaboración propia

Este sesgo geográfico va acompañado de un claro sesgo anglosajón a nivel idiomático y temático, puesto que una gran cantidad de los trabajos analizan casos concretos de bibliotecas anglosajonas. Para descartar que el sesgo pudiera estar producido por los términos de la cadena de búsqueda (en inglés), se repitió la misma búsqueda añadiendo los términos en español, y se pudo comprobar que el idioma añadido no aportaba ningún trabajo nuevo en los resultados.

Teniendo en cuenta que con la metodología de revisión utilizada se extraen los artículos más relevantes, destacados o con mayor impacto sobre el tema, su evolución puede tomarse como referencial de la evolución del tema a nivel general. A este respecto, a pesar de que el gráfico de dispersión de la distribución de los trabajos por año (figura 3) muestra un pico en 2013 y luego parece decaer, vemos que, realizando un diagrama de pareto (figura 4), el porcentaje del número de trabajos sobre el total (mostrado en la línea acumulativa) es claramente ascendente en la segunda mitad de los años analizados. Esto indica que el tema ha tenido una evolución continuada y considerable en los últimos años, y que el descenso del número de trabajos recopilados en los años 2016 y 2017, que se observa en el gráfico de dispersión, se debe más bien a la falta de tiempo hasta la fecha para que esos trabajos hayan tenido la repercusión necesaria como para que una revisión sistemática de literatura los incluyese entre los resultados. 


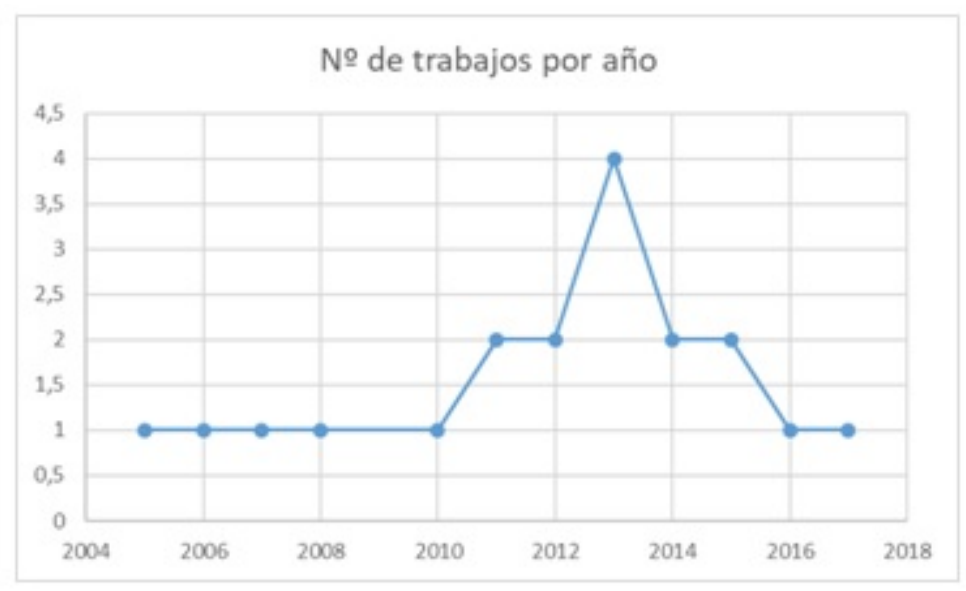

FIGURA 3

Gráfico de dispersión de la cantidad de trabajos recuperados en la revisión en relación con su año de publicación Elaboración propia.

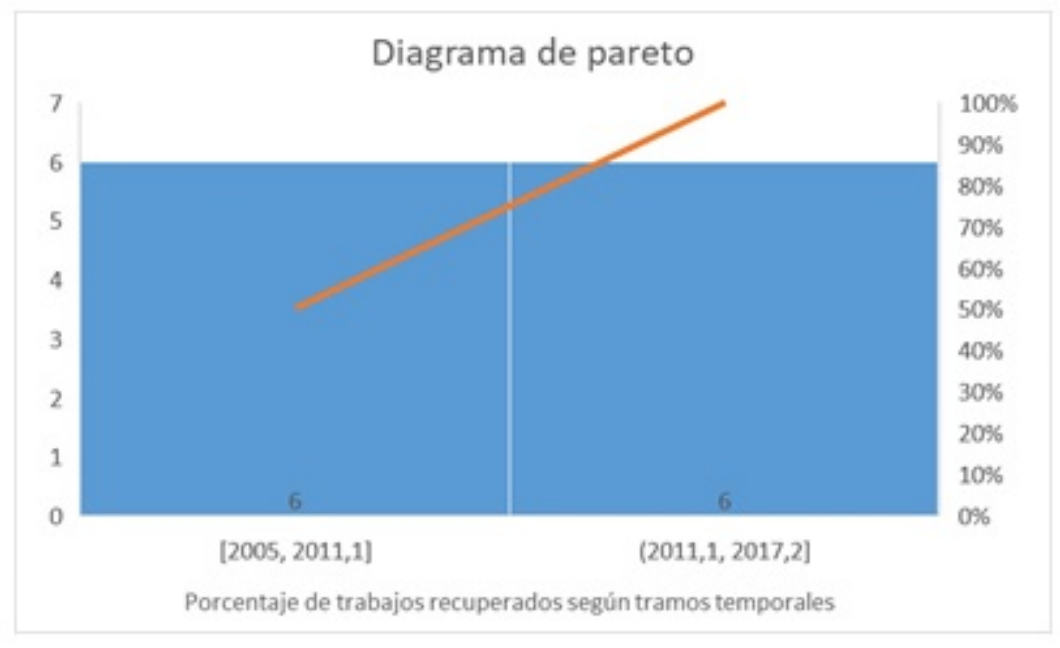

FIGURA 4

Diagrama de pareto mostrando la distribución de los trabajos en orden descendente de frecuencia en relación con el porcentaje del total mostrado en la línea acumulativa del eje secundario Elaboración propia

Otro detalle destacable es la notoria ausencia de palabras clave, siendo que sólo el $50 \%$ de los trabajos tienen asignadas palabras clave. Tanto si la ausencia depende de la base de datos, como si depende de la revista, se trata de una práctica poco recomendable, ya que perjudica a la visibilidad y recuperación de los artículos (Mangas-Vega, Dantas, Gómez-Díaz, y Merchán Sánchez-Jara, 2017).

Respecto a las palabras clave encontradas en el estudio, se distribuyen tal y como puede apreciarse en la figura 5. 


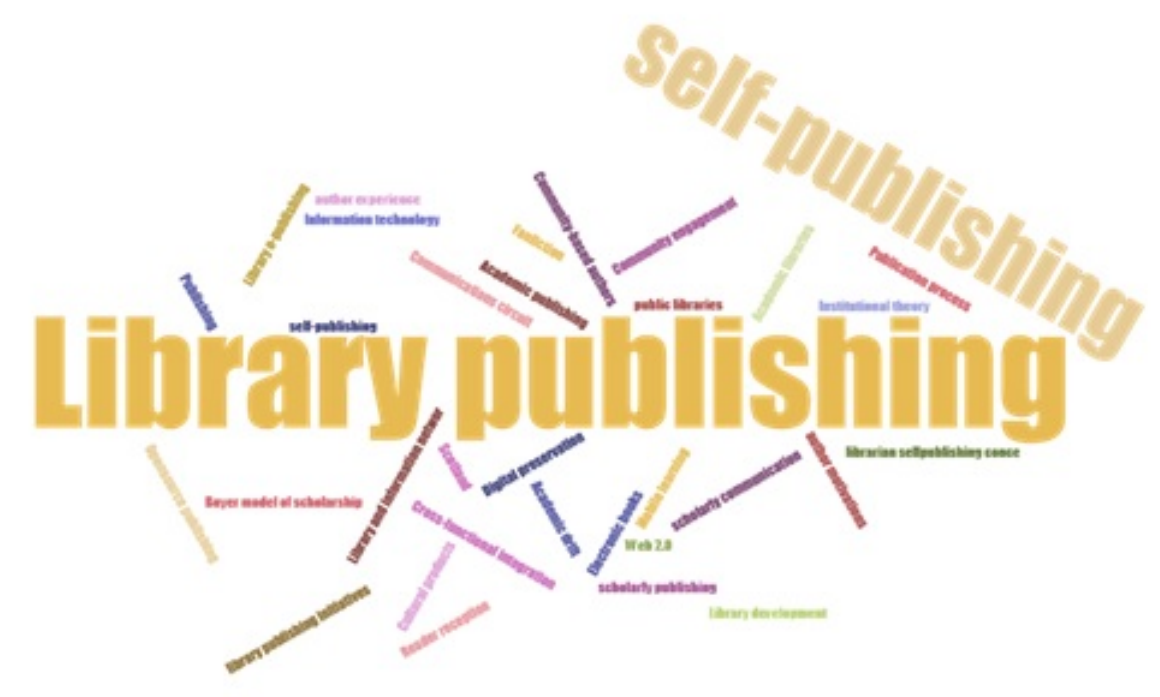

FIGURA 5

Nube de etiquetas de las palabras clave de los trabajos analizados en la SLR Elaboración propia.

Sólo un $10 \%$ de esas palabras clave hace alusión expresa a términos relacionados con el mundo digital y las nuevas tecnologías, un porcentaje muy bajo ya que los trabajos sí analizan mayoritariamente esos contextos. Quizás sea un indicativo de que este panorama (digital) empieza a ser considerado el estado habitual, y por tanto no necesita referencias ni alusiones específicas.

Avances respecto a la autopublicación en las bibliotecas:

La mayor parte de los proyectos y de los mayores avances en este tema se están desarrollando en USA. Y en todos los casos analizados, la tipología de esas bibliotecas es invariable: bibliotecas públicas, o académicas (también mayoritariamente públicas).

Se dan por parte de la biblioteca dos posiciones claramente diferenciadas: una que podría considerarse más pasiva, en la que el trabajo fundamental consiste en la revisión de las colecciones propias para comprobar si aparecen porcentajes adecuados de libros autopublicados; y otra posición que se podría considerar más activa, en la que la biblioteca ejerce de editora en sí misma o de soporte para usuarios-autores que quieren autopublicar.

Relacionando la tipología con la posición que adopta la biblioteca, se podría decir que las bibliotecas universitarias encajan mejor en lo que hemos denominado "posición activa"; llevan trabajando hace tiempo la parte de revisión de la colección, han desarrollado incluso herramientas y productos para salvar algunos problemas detectados (como la dificultad para buscar libros autopublicados, o la falta de metodología para preservar lo publicado) y por lo tanto ahora ya han dado un paso más y ejercen las funciones de editorial con proyectos y experiencias muy positivas. En alguno de los casos incluso se ha desarrollado un modelo para ayudar a otras bibliotecas que también quieran realizar esta función.

El tipo de autopublicación con el que trabajan las bibliotecas de los trabajos examinados es mayoritariamente digital. Sólo en contados casos se menciona el servicio de impresión bajo demanda.

Otra corriente a destacar entre los trabajos analizados es la que trata la autopublicación musical. En este caso, se está trabajando en desarrollar tres facetas principales por parte de la biblioteca: bibliotecacomo-editor, la publicación bibliotecaria y, también transversal a estas dos, la biblioteca como generadora de herramientas que ejerzan de filtro de calidad. Algo necesario dada la cantidad ingente de músicos que autopublican sus obras.

Por otra parte, existe un punto común reiterado en prácticamente la totalidad de los trabajos analizados: la necesaria formación de los bibliotecarios para poder atender las demandas y necesidades de los usuarios en esta 
materia. Las competencias necesarias son muchas y muy diversas, imposibles de abarcar por un bibliotecario si no recibe formación específica y especializada en el tema.

Algunas de las iniciativas y proyectos concretos que se analizan en los trabajos revisados, y que pueden servir de modelo para otras bibliotecas en todo el mundo, son los siguientes:

- The Woodneath Press, sistema desarrollado en la Woodneath Public Library que forma parte del sistema Mid-Continent Public Library (MCPL), y que se ubica en Kansas City (Missouri, USA). A través de este sistema, similar a un proveedor de servicios para autores, los usuarios que quieran autopublicar una obra reciben sesiones de formación en escritura e iniciativas de publicación, ayuda con la obtención del ISBN y apoyo para establecer otros derechos como el copyright o para atender todo el trabajo de marketing que implica la difusión y distribución de un libro autopublicado.

- Para poder dar a los bibliotecarios interesados formación especializada en la industria editora, algunos institutos como el University of Denver Publishing Institute, el New York University Center for Publishing's Summer Publishing Institute y el CUNY Publishing Institute, a través de la City University os New York Graduate School of Journalism, han promovido cursos especializados para su caso.

- La Topeka and Shawnee County Public Library trata a los autores que quieren autopublicar como propietarios de micronegocios, poniendo su foco en aumentar su acceso a los recursos y herramientas que necesiten para mejorar la calidad de sus productos (sus libros autopublicados).

- Otras bibliotecas como la Urbana Public Library de Illinois (USA), o la Seattle Public Library, han notado que el interés principal de sus usuarios que quieren autopublicar se centra en la historia y necesidades actuales de su localidad, por lo que, además de facilitarles la labor en la fase de producción, también han contribuido en la de difusión.

- Ejemplos similares de apoyo a la creación de elementos sobre la historia local a través de autopublicaciones los encontramos en la Bibliotech Digital Library de Bexar County en Texas (USA), con la colección "Recetas de mi vida”, y en la Alice Springs Public Library (Australia), con su colección Akaltye Antheme.

- En Los Gatos Public Library trabajaron para ayudar a publicar una antología poética de los alumnos del instituto local. La obra fue vendida a grandes distribuidores de e-books.

En todos los casos los resultados han sobrepasado las expectativas y las experiencias han sido muy positivas. Además de ayudar a los autores a publicar sus libros, han conseguido aumentar el interés de otros usuarios en servicios diferentes al préstamo de libros, e incluso se ha logrado generar un grato sentimiento de unidad en la comunidad alrededor de la biblioteca.

\section{Conclusiones}

La autopublicación como elemento integrado en las bibliotecas, ya sea como parte del catálogo ofrecido o como servicio prestado a los usuarios, es un tema de actualidad que a nivel científico está teniendo estudio y aplicación sostenidos en los últimos años.

A través de la distribución geográfica resultante de la revisión sistemática de literatura realizada, se ha podido comprobar que las bibliotecas estadounidenses son, sin duda alguna, las que más avanzada llevan la investigación sobre el tema. Se percibe en los trabajos su papel precursor en ofrecer servicios de publicación ya décadas atrás. Y el paso del tiempo y la llegada de las nuevas tecnologías no han supuesto una barrera, sino más bien un acicate para adaptarse a las nuevas necesidades de sus usuarios, reconvertir sus espacios, sus servicios y sistema de trabajo, generando a la vez nuevos modelos de biblioteca dignos de ser estudiados. El resto del mundo, sin embargo, y a tenor de los resultados obtenidos, parece situarse en un plano casi inexistente, excepto por algunos trabajos en bibliotecas de países de reminiscencias anglosajonas. Cabe preguntarse si no 
estarán siendo demasiado precavidos en un entorno vertiginosamente cambiante, de tal forma que cuando quieran introducirse en el tema la brecha sea ya insalvable.

Destacable es también el carácter mayoritariamente público de las bibliotecas en las que se está abordando este tema, sea cual sea la perspectiva que se le esté dando.

Otra conclusión que puede extraerse de la revisión es que las destrezas necesarias para llevar a buen puerto las iniciativas relacionadas con la autopublicación son muchas y muy variadas. Es necesaria una buena dotación presupuestaria que permita desarrollar las herramientas y productos necesarios para dar el servicio a los usuarios y que permita formar a los bibliotecarios en las competencias requeridas. A este respecto, es interesante destacar que prácticamente la totalidad de los trabajos revisados tratan proyectos e iniciativas desarrolladas en bibliotecas de carácter público, lo que implica una alta concienciación, a nivel gubernamental, sobre la relevancia de la autopublicación en el panorama actual del libro y sobre los buenos efectos que estas iniciativas pueden generar a nivel de satisfacción ciudadana y unidad de la comunidad.

Las experiencias son en todos los casos positivas, con muchas ventajas y apenas unos pocos inconvenientes.

La revisión realizada también ha permitido trazar líneas de trabajo futuro centradas en la investigación del tema en el mundo hispanohablante (con un notable incremento en títulos autopublicados en los últimos años). De dicha investigación podría surgir una línea de trabajo paralela que analizase las posibilidades de adaptación en otros países de alguno de los modelos utilizados en las bibliotecas norteamericanas, ya sean modelos de servicios editoriales dentro de las bibliotecas o sistemas de trabajo para la inclusión de obras autopublicadas en las colecciones de las mismas.

\section{REFERENCIAS}

Bowker (2014). Self-publishing in the United states, 2008-2013. Recuperado de http://media.bowker.com/documen ts/bowker_selfpublishinf_report2013.pdf

Cordón-García, J. A. (2011). La revolución del libro electrónico (vol. 2). Editorial UOC.

Cordón-García, J. A. (2016). Ruptura del campo editorial: la autopublicación y sus derivados. Anuario ThinkEPI, 10, 278-283.

EDI-RED (s.f.). Editores y Editoriales Iberoamericanos (siglos XIX-XXI). Recuperado de http://www.cervantesvirt ual.com/portales/editores_editoriales_iberoamericanos/

Furtado, J. A. (2012). Self-publishing: ¿tsunami o "business as usual"? En Cordón García, J.A. et al., Libros electrónicos $y$ contenidos digitales en la sociedad del conocimiento: Mercado, servicios y derechos (1st ed., pp. 69-92). Madrid: Pirámide.

Gómez-Díaz, R., García-Rodríguez, A., y Cordón-García, J. A. (2015). APPrender a leer y escribir: aplicaciones para el aprendizaje de la lectoescritura. Education in the Knowledge Society (EKS), 16(4), 118-137. Recuperado de h ttp://revistas.usal.es/index.php/revistatesi/article/view/eks2015164118137

Kitchenham, B. (2004). Procedures for performing systematic reviews. Keele, UK: Keele University.

Mangas-Vega, A. y Gómez-Díaz, R. (2015). Los criterios de calidad y la autopublicación. Anales de Documentación, 18(2). https://doi.org/10.6018/analesdoc.18.2.236541

Mangas-Vega, A. (2016a). Autopublicar. Los nuevos circuitos para autores e investigadores. Barcelona: UOC.

Mangas-Vega, A. (2016b). Los derechos del lector digital: revisión sistemática de la literatura. Métodos de Información, 7(13), 213-245. Disponible es: http://www.metodosdeinformacion.es/mei/index.php/mei/article/view/IIME I7-N13-213245/920

Mangas-Vega, A, Dantas, T., Gómez-Díaz, R. y Merchán Sánchez-Jara, J. (2017). Systematic Literature Reviews in Social Sciences and Humanities: a case study. Journal of Information Technology Research (JITR), 11(1).

Petticrew, M., y Roberts, H. (2008). Systematic reviews in the social sciences: A practical guide. Indianapolis: John Wiley $\&$ Sons. 
Ramírez, R. (2007). Calidad de vida relacionada con la salud como medida de resultados en salud: revisión sistemática de la literatura. Revista Colombiana de Cardiología, 14(4), 207-222.

Rodríguez Marcos, J. (9, junio, 2016). Muchos libros para pocos lectores. El País. Recuperado de https://elpais.com/ cultura/2016/06/07/actualidad/1465304358_275475.html

Saffle, M. (2010). Self-publishing and musicology\#: historical perspectives, problems, and possibilities. Notes, 66(4), 726-738. 